\title{
Relationship between lower limb torsion and coronal morphologies of the femur and tibia in patients with medial knee osteoarthritis
}

Shuntaro Nejima*, Yasushi Akamatsu, Hideo Kobayashi, Masaki Tsuji, Shota Mitsuhashi, Takahiro Sasaki, Ken Kumagai and Yutaka Inaba

\begin{abstract}
Background: To investigate the relationship between femoral or tibial torsion and hip-knee-ankle angle (HKA), mechanical lateral distal femoral angle (mLDFA), or mechanical medial proximal tibial angle (mMPTA) in patients with medial knee osteoarthritis (OA).

Methods: A total of 75 knees were enrolled. Femoral and tibial torsions were measured by superimposing the axial planes of computed tomography images. The relationship between femoral or tibial torsion and HKA, mLDFA, or mMPTA on radiographs was examined.

Results: The mean femoral torsion was $12.2 \pm 8.5^{\circ}$ internally; femoral internal and external torsions were observed in 70 and 5 knees, respectively. The mean tibial external torsion was $18.0 \pm 7.4^{\circ}$ externally; tibial external torsion was observed in all 75 knees. Femoral internal and tibial external torsions increased with lower mMPTA $(r=0.33, P=$ $0.003 ; r=-0.32, P=0.005$, respectively) but were not related to HKA or mLDFA.
\end{abstract}

Conclusion: Femoral and tibial torsions were correlated with varus inclination of the proximal tibia in patients with medial knee OA.

Keywords: Femoral and tibial torsions, Hip-knee-ankle angle, Knee osteoarthritis, Lateral distal femoral angle, Medial proximal tibial angle

\section{Background}

Femoral and tibial torsions can be measured using computed tomography $(\mathrm{CT})[1-25]$ and the values of patients with knee osteoarthritis $(\mathrm{OA})$ were reported $[2,3,6,12$, $14,16-18,21-24,26,27]$. The mean femoral internal and tibial external torsions evaluated by CT were $15.7^{\circ}$ to $19.7^{\circ}$ (range, $-6^{\circ}$ to $42^{\circ}$ ) and $11.3^{\circ}$ to $27.7^{\circ}$ (range, $-12^{\circ}$ to $34.8^{\circ}$ ) in medial knee OA, respectively [3, 12, 21-24]. The range of femoral and tibial torsions was wide and factors related

\footnotetext{
* Correspondence: nejishun@gmail.com

Department of Orthopaedic Surgery, Yokohama City University School of Medicine, 3-9 Fukuura, Kanazawa-ku, Yokohama 236-0004, Japan
}

to both torsions remain unclear. It is important to evaluate femoral and tibial torsions, because the relationship between these values and deformity of knee OA was reported $[2,22,23]$. However, CT images are not always obtained in clinical practice.

Tibial external torsion decreased with increasing varus inclination of medial tibial plateau in medial knee OA [22, 23]. As lower limb alignment changed from valgus to varus, tibial external torsion decreased in medial and lateral knee OA [2]. The only relationship between femoral or tibial torsions, and lower limb alignment or the proximal medial tibial deformity in knee OA has been investigated. If factors related to the 
femoral and tibial torsions were revealed, the values of femoral and tibial torsions will be predictable without CT images. Lower limb alignment is defined by the hip-knee-ankle angle (HKA), which is measured on long leg radiographs [28]. Of the anatomical elements of lower limb alignment, the present study focused on varus deformity of the distal femur and proximal tibia and investigated the relationship with femoral and tibial torsions. It was hypothesized that femoral and tibial torsions were related to the coronal deformity of the femur and tibia, respectively. The present study aimed to investigate the relationship between femoral or tibial torsion, and the coronal deformity of the femur and tibia in patients with medial knee OA.

\section{Methods}

A total of 57 female patients (75 knees) scheduled to undergo open wedge high tibial osteotomy (HTO) as treatment for medial knee OA from June 2011 to June 2016 were enrolled. The definition of knee OA was Kellgren-Laurence grade $\geq 2$ on anteroposterior standing radiographs. Radiographs and CT images were preoperatively obtained for the purpose of the surgery. Patients who had osteonecrosis of the knee, had hip or ankle OA, or underwent previous lower limb surgery were excluded. The mean knee flexion angle was $127.8 \pm 11.6^{\circ}$ (range, $100^{\circ}-145^{\circ}$ ) and extension angle was $-3.4 \pm 5.1^{\circ}$ (range, $-20^{\circ}-0^{\circ}$ ).

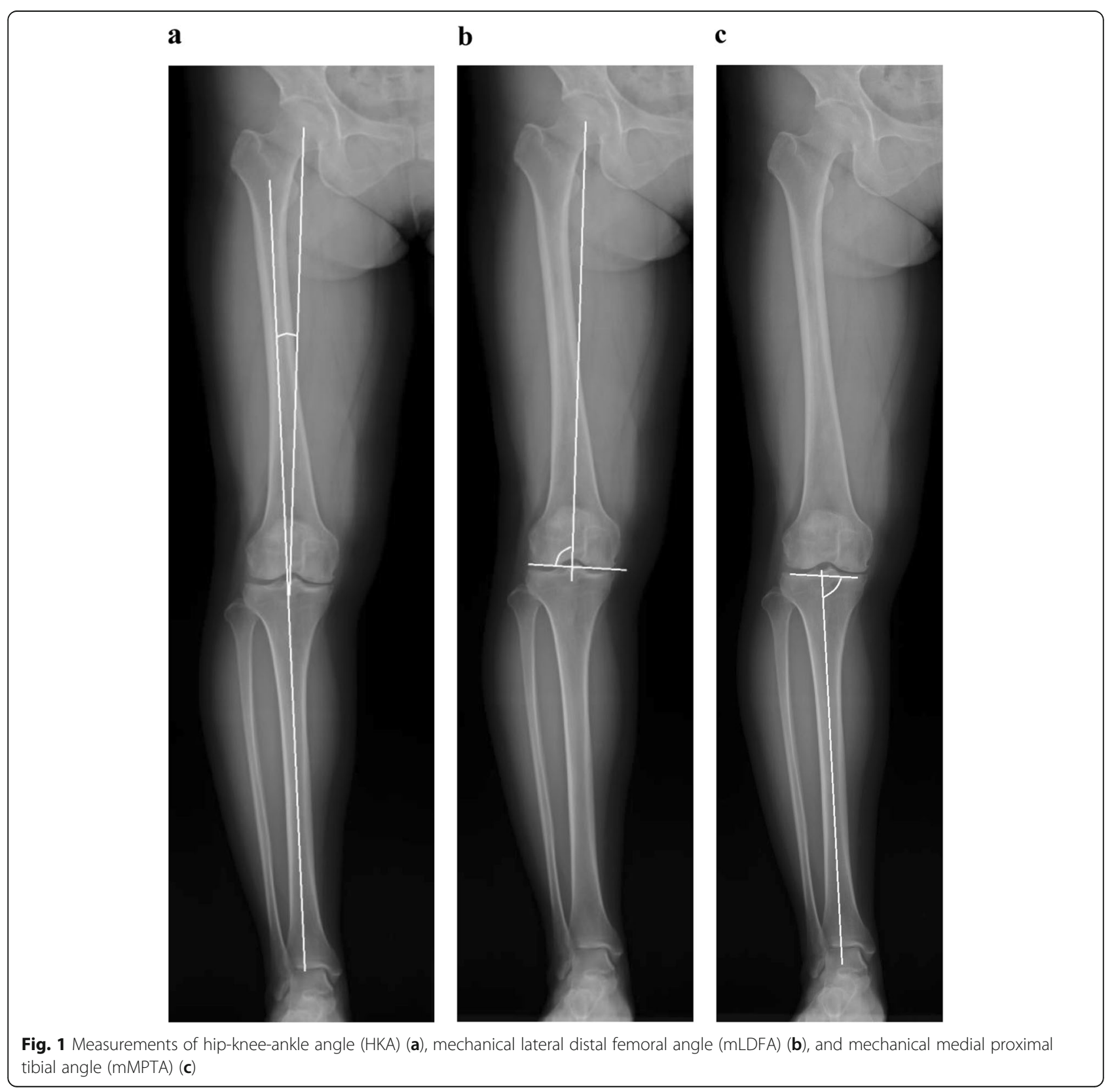



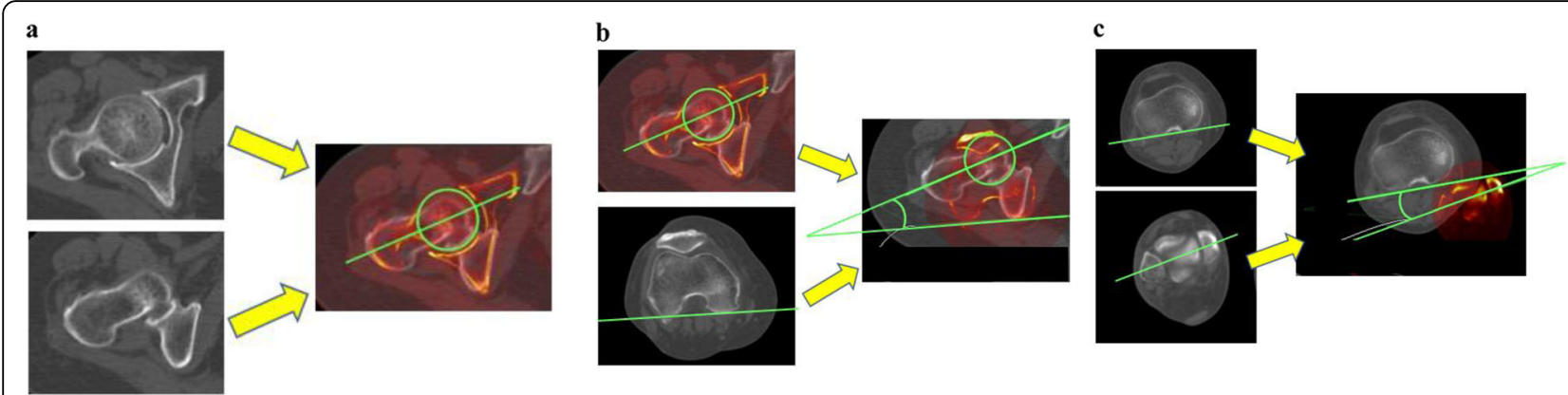

Fig. 2 a The proximal femoral torsional axis was assessed by superimposing two axial planes of the center of the femoral head and the central axis of the femoral neck. $\mathbf{b}$. Measurement of femoral torsion. Femoral torsion was defined as the angle between the proximal and distal femoral torsional axes. c. Measurement of tibial torsion. Tibial torsion was defined as the angle between the proximal and distal tibial torsional axes

Measurements of lower limb alignment and coronal morphologies of the distal femur and proximal tibia Anteroposterior whole-leg radiographs were obtained in one-leg standing position, with the knee joint maintained in extension. The lower limbs were positioned so that the patella faced forward and the X-ray beam centered on the knee. The radiographs obtained using a Fuji computed radiography system were projected, and HKA, mechanical lateral distal femoral angle (mLDFA), and mechanical medial proximal tibial angle (mMPTA) were measured using Fujifilm OP-A software (Fujifilm Co. Ltd., Tokyo, Japan) (Fig. 1a-c). HKA was defined as the angle between the mechanical axes of the femur and tibia; a positive value signified valgus alignment. Furthermore, mLDFA was defined as the lateral angle between the mechanical axis of the femur and the tangent to the femoral condyles, and MMPTA was defined as the medial angle between the mechanical axis of the tibia and the joint line of the proximal tibia. The mLDFA and mMPTA represent the coronal deformity of the femur and tibia, respectively. Normal values for each of the radiological parameters reported in literature are $85^{\circ}-90^{\circ}$ for mLDFA and MMPTA [29].

\section{Measurement of femoral torsion}

With patients lying supine, whole-leg CT images were acquired using 1.5-mm-thick slices obtained on a

Table 1 Patient demographic characteristics

\begin{tabular}{ll}
\hline Knees, $\mathrm{n}$ & 75 \\
Age, $\mathrm{yr}$ & $64.9 \pm 7.7(46-78)$. \\
Height, cm & $154.8 \pm 5.4(138.5-172.1)$ \\
Weight, kg & $60.8 \pm 9(43.8-85)$ \\
Body mass index & $25.4 \pm 3.6(19-33.9)$ \\
Side, left/right & $37 / 38$ \\
Kellgren-Laurence grade 3/4 & $37 / 38$ \\
Ahlbäck grade 1/2/3 & $58 / 12 / 5$ \\
\hline
\end{tabular}

Data are presented mean \pm standard deviation with range in the parenthesis
SOMATOM Sensation 16 CT scanner (Siemens, Munich, Germany).

Femoral and tibial torsions were measured using OsiriX software (Pixmeo, Genova, Switzerland), which enabled superimposition of axial planes of CT images. Femoral torsion was assessed using the method described by Reikerås et al. [20]. We initially selected axial planes of the center of the femoral head and the central axis of the femoral neck, and subsequently superimposed these planes. The proximal femoral torsional axis was defined as the line through the center of the femoral head and the central axis of the femoral neck (Fig. 2a). The distal femoral torsional axis was the line connecting most posterior edge of the femoral condyles. Femoral torsion was defined as the angle between the proximal and distal femoral torsional axes (Fig. 2b). If the distal torsional axis was external relative to the proximal torsional axis, the torsion angle was recorded as a positive value.

\section{Measurement of tibial torsion}

Tibial torsion, which was assessed using the bimalleolar method [15], was defined as the angle between the proximal and distal tibial torsional axes. The proximal tibial torsional axis was defined as the line tangent to the posterior contour of the proximal tibia above the

Table 2 Radiographic and CT data

\begin{tabular}{ll}
\hline HKA & $-8.8 \pm 3.4^{\circ}\left(-0.5^{\circ}--16.4^{\circ}\right)$ \\
mLDFA & $88.4 \pm 2.6^{\circ}\left(83.1^{\circ}-95.5^{\circ}\right)$ \\
mMPTA & $84.4 \pm 2.5^{\circ}\left(78.9^{\circ}-89.0^{\circ}\right)$ \\
Femoral internal torsion & $12.2 \pm 8.5^{\circ}\left(-6.5^{\circ}-28.9^{\circ}\right)$ \\
Tibial external torsion & $18.0 \pm 7.4^{\circ}\left(2.6^{\circ}-37.8^{\circ}\right)$ \\
Sum of femoral and tibial torsions & $5.9 \pm 11.2^{\circ}\left(-19.8^{\circ}-39.0^{\circ}\right)$
\end{tabular}

Data are presented mean \pm standard deviation with range in the parenthesis. The mLDFA, mechanical lateral distal femoral angle; mMPTA, mechanical medial proximal tibial angle; HKA, hip-knee-ankle angle. A positive value means valgus alignment 

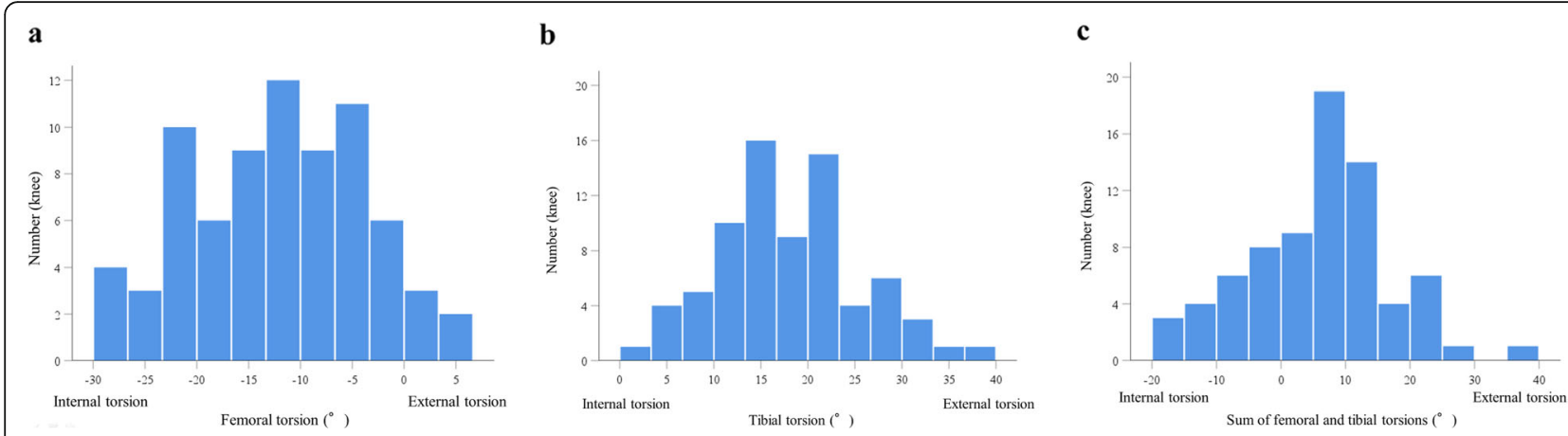

Fig. 3 a. Histogram of femoral torsion. b. Histogram of tibial torsion. c. Histogram of sum of femoral and tibial torsions

proximal end of the fibula. The distal tibial torsional axis was defined as the line through the midpoints of the articular surfaces of the medial and lateral malleoli (Fig. 2c). If the distal torsional axis was external relative to the proximal torsional axis, the torsion angle was recorded as a positive value. All parameters on radiographs and $\mathrm{CT}$ images were measured by two experienced orthopaedic surgeons (SN, YA).

\section{Statistical analysis}

Data were expressed as mean \pm standard deviation and normal distribution was confirmed using the Shapiro-Wilk test. Simple linear regression (Pearson correlation coefficient) was used to examine the relationship between femoral or tibial torsion, and HKA, mLDFA or MMPTA, and the relationship between femoral and tibial torsions. All statistical analyses were performed using IBM SPSS for Windows version 21.0 (IBM Corporation, Armonk, NY, USA). P values $<0.05$ were considered statistically significant. A power analysis was performed on correlations $(r=$ 0.5 , significance level $=0.05$, power $=0.80$ ) using G*Power version 3.1.9.2 (Heinrich-Heine-Universität Düsseldorf, Germany). A priori power analysis resulted in a sample size of 26 . To examine the reproducibility of each measurement, we randomly selected 20 knees, and intraclass correlation coefficient $(\operatorname{ICC})(1,1)$ and $\operatorname{ICC}(2,1)$ were calculated for intra- and inter-observer reliabilities, respectively. The scoring system of Landis and Koch [30] was used to analyze the results-that is, almost perfect, $>0.81$; substantial, $0.61-0.80$; moderate, 0.41-0.60; fair, 0.21-0.40; and slight, 0.0-0.20.

\section{Results}

The demographic data are shown in Table 1, whereas the radiographic and CT data are summarized in Table 2. The mean femoral internal torsion was $12.2 \pm 8.5^{\circ}$; femoral internal and external torsions were observed in 70 and 5 knees, respectively (Fig. 3a).
The mean tibial external torsion was $18.0 \pm 7.4^{\circ}$; tibial external torsion was observed in all 75 knees (Fig. 3b). The mean sum of femoral and tibial torsions was $5.9 \pm 11.2^{\circ}$ (Fig. 3c). Femoral internal and tibial external torsions increased with lower mMPTA (femoral internal torsion: $r=0.33, P=0.003$; tibial external torsion: $r=-0.32, P=0.005)$. No relationship was observed between both torsions and HKA or mLDFA (Fig. 4a-f, Table 3). Femoral torsion was not related to tibial torsion $(r=0.00, P=0.98$ [Fig. 4g]). The intra- and inter-observer ICC values were 0.94 (95\% CI $0.85-0.97)$ and $0.92(0.76-0.97)$ for femoral torsion, $0.92(0.82-0.97)$ and $0.91(0.80-0.97)$ for tibial torsion, $0.99(0.97-0.99)$ and $0.98(0.84-0.99)$ for HKA, $0.89(0.75-0.96)$ and 0.89 (0.75-0.95) for mLDFA, and $0.89(0.74-0.95)$ and 0.81 (0.58-0.92) for mMPTA, respectively. All measured intraand inter-observer ICCs were almost perfect according to the scoring system of Landis and Koch [30].

\section{Discussion}

The important findings of the present study were that femoral internal and tibial external torsions increased with lower mMPTA, but were not related to HKA or mLDFA in patients with medial knee OA. In addition, femoral internal and tibial external torsions neutralized each other, but were not correlated.

With respect to tibial torsion and loading distribution on the tibia, Yazdi et al. [31] investigated the relationship between tibial torsion and medial and lateral compartment contact pressure in the knee joint using cadaveric specimens. They reported that a $15^{\circ}$ external change in tibial torsion decreased the medial compartment contact pressure and increased the lateral compartment contact pressure. In contrast, a $15^{\circ}$ internal change in tibial torsion increased the medial compartment contact pressure and decreased the lateral compartment contact pressure. In the present study, tibial external torsion increased with lower mMPTA. This might be a compensation mechanism for the varus deformity of the tibia. 
a

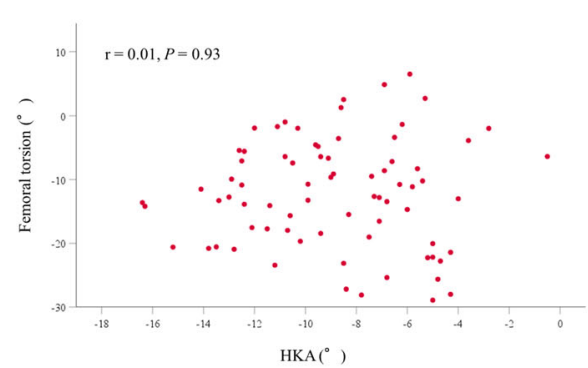

b

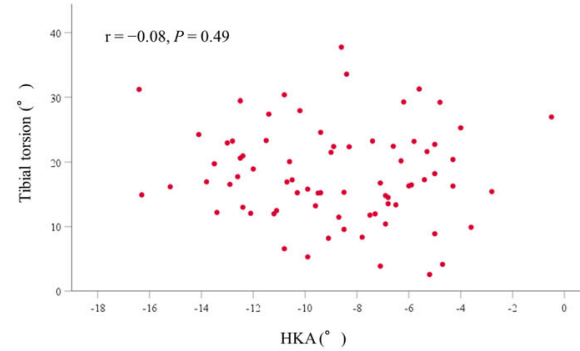

c

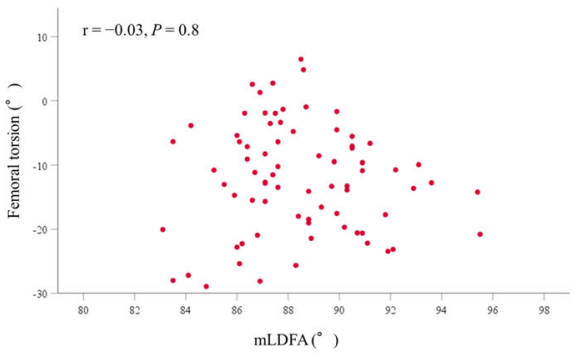

d

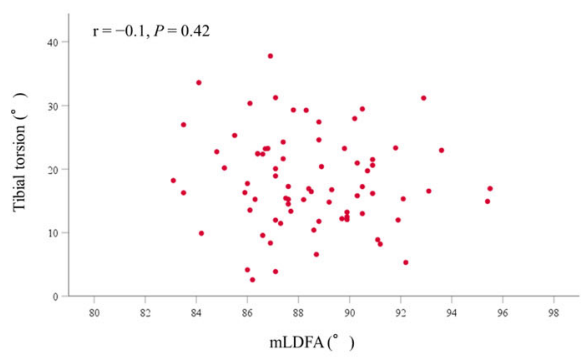

e

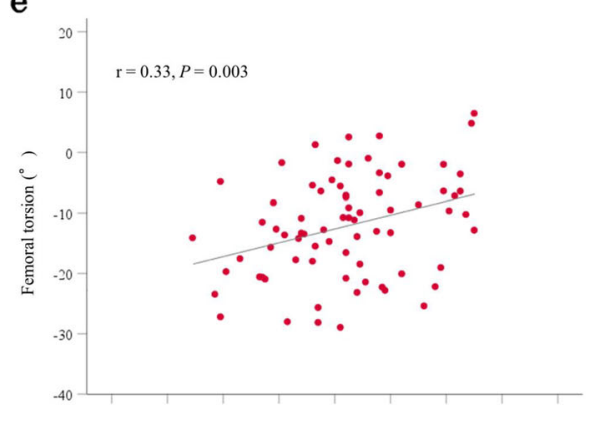

f

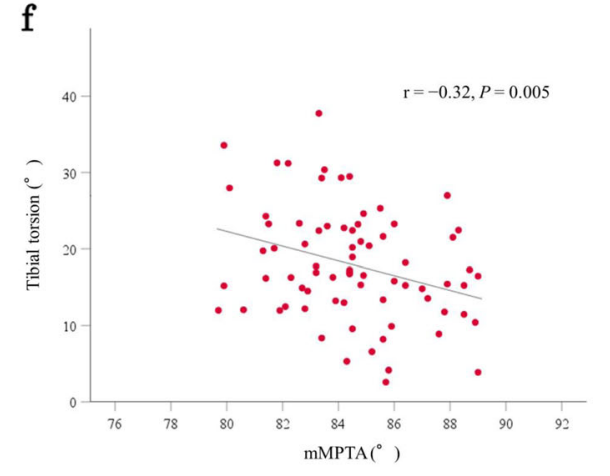

g

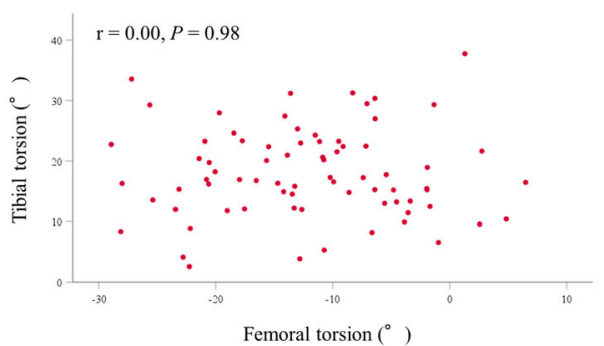

Fig. 4 a. Relationship between hip-knee-ankle angle (HKA) and femoral torsion. b. Relationship between hip-knee-ankle angle (HKA) and tibial torsion. c. Relationship between mechanical lateral distal femoral angle (mLDFA) and femoral torsion. d. Relationship between mechanical lateral distal femoral angle (mLDFA) and tibial torsion. e. Relationship between mechanical medial proximal tibial angle (mMPTA) and femoral torsion. Femoral torsion increased with higher mMPTA. f. Relationship between mechanical medial proximal tibial angle (mMPTA) and tibial torsion. Tibial torsion increased with lower MMPTA. g. Relationship between femoral and tibial torsions

Interestingly, femoral internal torsion increased with lower mMPTA in the present study. We speculate that the relationship between femoral internal torsion and lower mMPTA might have restored the change in joint line obliquity in continuous flexed knee position. In the case with femoral internal torsion and normal mMPTA, the tibial axis in the coronal plane will change to valgus if the femur is fixed and the knee is flexed. Internal torsion of the posterior medial femoral condyle has a tendency to move the medial 
Table 3 Relationship between femoral and tibial torsions and HKA, mLDFA, and MMPTA

\begin{tabular}{lllll}
\hline & Femoral torsion & $P$ & Tibial torsion & $P$ \\
\hline HKA & $r=0.01$ & 0.93 & $r=-0.08$ & 0.49 \\
mLDFA & $r=-0.03$ & 0.8 & $r=-0.1$ & 0.42 \\
mMPTA & $r=0.33$ & 0.003 & $r=-0.32$ & 0.005
\end{tabular}

HKA, hip-knee-ankle angle; mLDFA, mechanical lateral distal femoral angle; mMPTA, mechanical medial proximal tibial angle

joint line down in flexed position. Therefore, the relationship between femoral internal torsion and lower mMPTA shows a physiological interaction to adjust the change in joint line obliquity and coronal tibial alignment in flexed position. Moreover, femoral internal torsion and lower mMPTA might enable the joint line to be parallel to the ground in continuous flexed position.

Femoral internal torsion was correlated with tibial external torsion in normal cadaveric specimens in a previous study [32]. Meanwhile, femoral internal torsion was not correlated with tibial external torsion in the present study. Duparc et al. [3] evaluated the sum of femoral and tibial torsions as the index of cumulative torsions (ICT). They reported that ICT was highly variable and there were three morphotypes of ICT in patients with medial knee OA. In accordance with their result, ICT was highly variable in the present study. This might lead to no correlation between femoral and tibial torsions in the present study. On the other hand, mMPTA was correlated with both femoral and tibial torsions in the present study. The clinical relevance of this study is that the values of femoral and tibial torsions can be predictable from the varus inclination of the proximal tibia on radiographs in patients with medial knee OA. Although correlation coefficients in this study were not high and further studies are needed, this study may be helpful for the prediction of femoral and tibial torsions on radiographs in future.

The present study has a few limitations. First, the present results were obtained from Japanese female patients. Whether these results can be generalized to male patients is unclear. We excluded male patients in consideration of significant sex difference in femoral torsion among subjects with medial knee OA [17]. However, the prevalence of knee OA was higher in female than in male in Japanese population [33]. Thus, the result of the present study can be applicable to many patients in Japan. Second, the extent of OA in patients in this study was classified as Ahlb ck grade 1 to 3 . Whether mMPTA is related to femoral and tibial torsions in a healthy population or in patients with more severe osteoarthritic knees is unknown. Third, the lower limb position on $\mathrm{CT}$ scan and flexion contracture may affect the measured value on CT images. However, the measurement of femoral and tibial torsions using $\mathrm{CT}$ was not affected by position when the femorotibial angle was less than $195^{\circ}$ and flexion contracture was less than $20^{\circ}$ [21]. Every patient in the present study underwent open wedge $\mathrm{HTO}$, and the range of knee extension angle was $-20^{\circ}-0^{\circ}$. Our indications for open wedge HTO include femorotibial angle less than $185^{\circ}$. Therefore, the measurement of femoral and tibial torsions was less influenced by the lower limb position and flexion contracture. Fourth, femoral and tibial torsions were measured in the supine position, whereas HKA, mLDFA, and MMPTA were measured on standing radiographs. This is a retrospective study and one-leg standing radiographs were taken for the surgical planning. Anteroposterior standing radiographs could increase joint line convergence angle and affect HKA, compared with supine radiographs. However, mLDFA and MMPTA might be less affected by anteroposterior standing radiographs.

\section{Conclusion}

Femoral and tibial torsions were correlated with varus inclination of the proximal tibia in patients with medial knee OA.

\section{Abbreviations \\ CT: Computed tomography; OA: Osteoarthritis; HKA: Hip-knee-ankle angle; mLDFA: mechanical Lateral distal femoral angle; mMPTA: mechanical Medial proximal tibial angle; HTO: High tibial osteotomy; ICCs: Intraclass correlation coefficients; ICT: Index of cumulative torsions}

\section{Acknowledgements \\ None.}

Authors' contributions

SN: study design, data collection, interpreting the data and writing the paper. YA, HK: data collection. MT, SM, TS, KK, YI: interpretation. All authors read and approved the final manuscript.

\section{Funding}

No funding was obtained for this study.

\section{Availability of data and materials}

The datasets used and/or analyzed during the current study available from the corresponding author on reasonable request.

Ethics approval and consent to participate

Ethical approval for the study was obtained from the institutional review board of Yokohama City University Hospital (B150108021). Informed and written consent was obtained from all patients.

Consent for publication

Not applicable.

Competing interests

The authors declare that they have no competing interests.

Received: 27 November 2019 Accepted: 13 April 2020

Published online: 17 April 2020

References

1. Buck F, Guggenberger R, Koch P, Pfirrmann C. Femoral and tibial torsion measurements with 3D models based on low-dose biplanar radiographs in comparison with standard CT measurements. AJR. 2012;199:607-12. 
2. Chang MJ, Jeong HJ, Kang SB, Chang CB, Yoon C, Shin JY. Relationship between coronal alignment and rotational profile of lower extremity in patients with knee osteoarthritis. J Arthroplast. 2018;33:3733-77.

3. Duparc F, Thomine JM, Simonet J, Biga N. Femoral and tibial bone torsions associated with medial femoro-tibial osteoarthritis. Index of cumulative torsions. Orthop Traumatol Surg Res. 2014;100:69-74.

4. Eckhoff DG, Johnson KK. Three-dimensional computed tomography reconstruction of tibial torsion. Clin Orthop Relat Res. 1994:302:42-6.

5. Eckhoff DG, Johnston RJ, Stamm ER, Kilcoyne RF, Wiedel JD. Version of the osteoarthritic knee. J Arthroplast. 1994;9:73-9.

6. Enomoto H, Nakamura T, Waseda A, Niki Y, Toyama Y, Suda Y. A novel and reproducible reference axis for distal tibial axis rotation. J Arthroplast. 2013;28:788-91.

7. Folinais D, Thelen P, Delin C, Radier C, Catonne Y, Lazennec JY. Measuring femoral and rotational alignment: EOS system versus computed tomography. Orthop Traumatol Surg Res. 2013;99:509-16.

8. Hernandez RJ, Tachdjian MO, Poznanski AK, Dias LS. CT determination of femoral torsion. AJR. 1981;137:97-101.

9. Høiseth $A$, Reikerås $O$. Fønstelien. Evaluation of three methods for measurement of femoral neck anteversion. Acta Radiol. 1988:30:69-73.

10. Jakob RP, Haertel M, Stüssi E. Tibial torsion calculated by computed tomography and compared to other methods of measurement. J Bone Joint Surg. 1980;62B:238-42.

11. Jend HH, Heller M, Dallek M, Schoettle H. Measurement of tibial torsion by computer tomography. Acta Radiol Diagn. 1981;22:271-6.

12. Khan MS, Seon JK, Song EK. Rotational profile of lower limb and axis for tibial component alignment in varus osteoarthritic knees. J Arthroplast. 2012;27:797-802

13. Kristiansen LP, Gunderson RB, Steen H, Reikerås O. The normal development of tibial torsion. Skelet Radiol. 2001;30:519-22.

14. Laasonen EM, Jokio P, Lindholm TS. Tibial torsion measured by computed tomography. Acta Radiol Diagn. 1984;25:325-9.

15. Liodakis E, Doxastaki I, Chu K, Krettek C, Gaulke R, Citak M, et al. Reliability of the assessment of lower limb torsion using computed tomography: analysis of five different techniques. Skelet Radiol. 2012;41:305-11.

16. Mochizuki T, Tanifuji O, Koga Y, Hata R, Mori T, Nishino K, et al. External torsion in a proximal tibia and internal torsion in a distal tibia occur independently in varus osteoarthritic knees compared to healthy knees. J Orthop Sci. 2017:22:501-5.

17. Mochizuki T, Tanifuji O, Koga Y, Sato T, Kobayashi K, Nishino K, et al. Sex difference in femoral deformity determined using three-dimensional assessment for osteoarthritic knees. Knee Surg Sports Traumatol Arthrosc. 2017;25:468-76

18. Moussa M. Rotational malalignment and femoral torsion in osteoarthritic knees with patellofemoral joint involvement. Clin Orthop Relat Res. 1994:304:176-83.

19. Nagamine R, Miyanishi K, Miura H, Urabe K, Matsuda S, Iwamoto Y. Medial torsion of the tibia in Japanese patients with osteoarthritis of the knee. Clin Orthop Relat Res. 2003:408:218-24.

20. Reikerås O, Bjerkreim I, Kolbenstvedt A. Anteversion of the acetabulum and femoral neck in normals and in patients with osteoarthritis of the hip. Acta Orthop Scand. 1983;54:18-23.

21. Takai S, Sakakida K, Yamashita F, Suzu F, Izuta F. Rotational alignment of the lower limb in osteoarthritis of the knee. Int Orthop. 1985;9:209-16.

22. Yagi T. A study on torsion of the lower leg in osteoarthritis of the knee (in Japanese). J Jpn Orthop Ass. 1984;58:405-17.

23. Yagi T. Tibial torsion in patients with meial-type osteoarthrotic knees. Clin Orthop Relat Res. 1994;302:52-6.

24. Yagi T, Sasaki T. Tibial torsion in patients with medial-type osteoarthritic knee. Clin Orthop Relat Res. 1986;213:177-82.

25. Yin $Y$, Zhang $L$, Hou Z, Yang Z, Zhang $R$, Chen W, et al. Measuring femoral neck torsion angle using femoral neck oblique axial computed tomography reconstruction. Int Orthop. 2016;40:371-6.

26. Turner MS. The association between tibial torsion and knee joint pathology. Clin Orthop Relat Res. 1994;302:47-51.

27. Turner MS, Smillie IS. The effect of tibial torsion on the pathology of the knee. J Bone Joint Surg. 1981;63B:396-8

28. Cooke D, Scudamore A, Li J, Wyss U, Bryant T, Costigan P. Axial lower-limb alignment: comparison of knee geometry in normal volunteers and osteoarthritis patients. Osteoarthr Cart. 1997;5(1):39-47.

29. Paley D, Herzenberg JE, Tetsworth K, McKie J, Bhave A. Deformity planning for frontal and sagittal plane corrective osteotomies. Orthop Clin North Am. 1994;25:425-65
30. Landis JR, Koch GG. The measurement of observer agreement for categorical data. Biometrics. 1977:33:159-74.

31. Yazdi $H$, Mallakzadeh M, Farshidfar SS, Givehchian B, Daneshparvar $H$, Behensky $\mathrm{H}$. The effect of tibial rotation on knee medial and lateral compartment contact pressure. Knee Surg Sports Traumatol Arthrosc. 2016; 24:79-83.

32. Kobyliansky E, Weissman SL, Nathan H. Femoral and tibial torsion. Int Orthop. 1979;3:145-7.

33. Yoshimura N, Muraki S, Oka H, Mabuchi A, En-Yo Y, Yoshida M, et al. Prevalence of knee osteoarthritis, lumbar spondylosis, and osteoporosis in Japanese men and women: the research on osteoarthritis/osteoporosis against disability study. J Bone Miner Metab. 2009;27:620-8.

\section{Publisher's Note}

Springer Nature remains neutral with regard to jurisdictional claims in published maps and institutional affiliations.
Ready to submit your research? Choose BMC and benefit from:

- fast, convenient online submission

- thorough peer review by experienced researchers in your field

- rapid publication on acceptance

- support for research data, including large and complex data types

- gold Open Access which fosters wider collaboration and increased citations

- maximum visibility for your research: over $100 \mathrm{M}$ website views per year

At $\mathrm{BMC}$, research is always in progress.

Learn more biomedcentral.com/submissions 